\title{
Dynamic Analysis and Design of a Balancer for a Three-Column Centrifuge
}

\author{
Hai-Wei Chen ${ }^{1,2}$ and Qiu-Ju Zhang ${ }^{1,2}$ \\ ${ }^{1}$ School of Mechanical Engineering, Jiangnan University, No. 1800 Lihu Avenue, Wuxi, Jiangsu 214122, China \\ ${ }^{2}$ Jiangsu Key Laboratory of Advanced Food Manufacturing Equipment and Technology, Wuxi, Jiangsu 214122, China \\ Correspondence should be addressed to Hai-Wei Chen; chenhaiwei8012@163.com
}

Received 26 May 2015; Accepted 27 August 2015

Academic Editor: Francesco Ripamonti

Copyright (c) 2016 H.-W. Chen and Q.-J. Zhang. This is an open access article distributed under the Creative Commons Attribution License, which permits unrestricted use, distribution, and reproduction in any medium, provided the original work is properly cited.

This paper studies dynamics of a three-column centrifuge and tries to design a ball balancer to suppress its vibration and increase its operating speed. At first, two vibration models with and without the balancer are introduced. Then modal analysis of the centrifuge itself is carried out with two isolated natural frequency groups identified. Centrifugal forces acting on the system at the steady state are analyzed and a tip-over phenomenon is discovered and validated through simulations. A ball balancer is adopted in the centrifuge and several potential tip-over phenomena related to the balancer are studied. At last, a comparison between dynamics of the centrifuge before and after the balancer is installed is made with benefits and side effects of the balancer presented.

\section{Introduction}

A three-column centrifuge is named due to its three sup porting legs. Up until now, it has been widely employed in industries of textile, food, pharmacy, chemistry, metallurgy, and so forth, for the purpose of dehydration, incrassation, separation, classification of solid particles, and so forth.

Researches on the design of the three-column centrifuge can be found as early as the 1960s. For satisfying organization assembly line production at chemical plants, Gerke and Noskov [1] discussed the shearing off and discharge of the centrifuged cake. Then they improved the centrifuge by employing a hydraulic motor [2]. In 1968, a continuous centrifuge in the metal working industry was introduced by Il'yashenko and Gerke [3] for continuous deoiling of metal chips contaminated by oil.

Some early studies on dynamics of the centrifuge can be found in the late 1960s. A linear vibration model was built by Likuchev [4] assuming that vibration amplitude of the centrifuge is quite small, and then frequencies of the centrifuge were studied. Later, the rigidity of several types of supports was discussed in [5]. The steady motion of the centrifuge was investigated by Vanichkin [6] with the reaction force of the suspension system calculated. However, most of these studies were based on linear models, and nonlinear dynamic characteristics of the centrifuge were not presented. Serious vibrations often happen once the centrifuge operates above a certain speed. Up until now, not much work has been done on the reason and a solution to this is still unclear.

The suspension structure of the centrifuge is quite similar to that of the vertical axis washing machine with a pendulant suspension system [7-11]. Thus, the balancer demonstrated in the washer may also be employed in the centrifuge. However, differences between the centrifuge and the washer have to be considered. The biggest difference lies in the stiffness of their suspension systems. The centrifuge employs rigid suspension rods while the washer uses soft springs. Thus, the suspension system of the centrifuge is much stiffer than that of the washer. Although this makes complete balance of the centrifugal forces much easier, side effects could be induced such as the tip-over phenomenon discovered in this paper.

The balancer has been studied for many years. When the rotational speed of a rotor is much higher than its critical frequency, eccentricity of the system can be partially or sometimes totally counteracted by the balancer. However, disastrous consequences can still happen even after the 


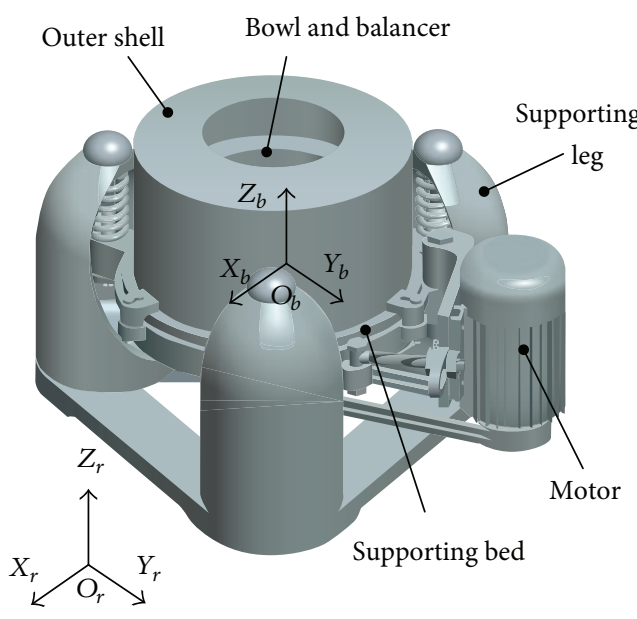

(a)

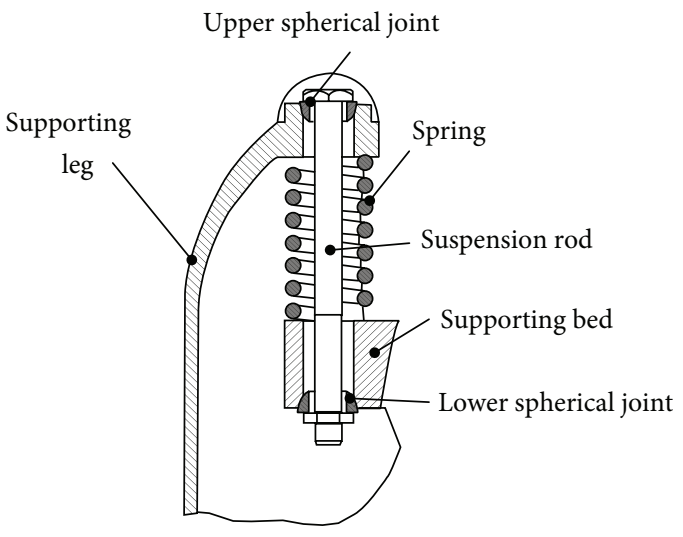

(b)

Figure 1: A three-column centrifuge (a) and its suspension structure (b).

eccentricity is eliminated, especially when the eccentricity and the balancer are in different planes. The three-column centrifuge provides a very good example of such condition. The tip-over phenomenon can still occur even after the elimination of the eccentricity.

The structure of this paper is as follows. Vibration models of the centrifuge were introduced in Section 2. Then dynamics of the centrifuge itself are studied in Section 3.1. Influences of the balancer on dynamics of the centrifuge are discussed in Section 3.2. And a comparison between dynamics of the centrifuge before and after the balancer is installed is made in Section 3.3. At last, some conclusions are drawn in Section 4.

\section{Vibration Models of the Three-Column Centrifuge}

As can be seen from Figure 1(a), a three-column centrifuge mainly comprises a core assembly and a suspension system. The assembly is composed of a bowl, an outer shell, a supporting bed, and a motor. The motor and the outer shell are fixed on the supporting bed, while the bowl is installed on the bed through a revolving pivot. Figure 1(b) shows the suspension structure of the centrifuge; as can be seen, the supporting bed is connected to the supporting legs though three suspension rods with spherical joints at both ends. A compression spring is inserted between each leg and the supporting bed.

For describing movements of the core assembly, two reference frames are constructed in Figure 1: a global one $X_{r} Y_{r} Z_{r}$ on the ground and a dynamic one $X_{b} Y_{b} Z_{b}$ in the core assembly.

The structure of the three-column centrifuge is quite similar to that of the vertical axis washing machine discussed in [7-11]. And the vibration model of the centrifuge can be similarly constructed using Lagrange's equation based on the kinetic and geopotential energies derived in $[8,11]$; thus, only the final forms of the vibration models are summarized here.
Two models with and without the balancer are built in the paper. The one without balancer can be expressed as

$$
\begin{aligned}
{[\mathbf{M}] \ddot{\mathbf{q}}=} & \frac{1}{2}\left[\frac{\partial[\mathbf{M}]}{\partial \mathbf{q}} \dot{\mathbf{q}}\right]^{T} \dot{\mathbf{q}}-[\dot{\mathbf{M}}] \dot{\mathbf{q}}+\mathbf{F}\left(\dot{\theta}_{m}, \ddot{\theta}_{m}\right)+\mathbf{Q} \\
& -\frac{\partial V_{g}}{\partial \mathbf{q}}
\end{aligned}
$$

where $\mathbf{q}=\left[\begin{array}{llllll}x & y & z & \alpha & \beta & \gamma\end{array}\right]^{T}$ with $\left\{\begin{array}{lll}x & y & z\end{array}\right\}$ denoting the translational displacement of $O_{b}$ (the origin of the dynamic reference frame $X_{b} Y_{b} Z_{b}$ ) and $\{\alpha \beta \gamma\}$ being the Cardan angles; $\mathbf{F}\left(\dot{\theta}_{m}, \ddot{\theta}_{m}\right)$ is a vector relative to the rotational speed $\dot{\theta}_{m}$ and rotational acceleration $\ddot{\theta}_{m}$ of the motor; $\mathbf{Q}$ is the generalized forces provided by the suspension system; $[\mathbf{M}]$ and $V_{g}$ are the mass matrix and geopotential energy of the core assembly. A detailed expression of the above model can be found in [11].

For suppressing vibrations of the centrifuge, a balancer composed of a circular cavity and several balls is employed. The cavity is fixed on the bowl and the balls can run freely in the cavity. For exploring basic characteristics of the dynamic system, the balancer is assumed to be ideally manufactured and lubricated, and only viscous damping exists between the balls and the cavity; besides, a two-ball model is employed and the idea that no collision happens between the balls is assumed. Suppose that the rotational angle of the $i$ th ball relative to the cavity (or bowl) is $\phi_{i}$; the vibration model of the centrifuge with a ball balancer can be described as

$$
\begin{aligned}
{\left[\mathbf{M}_{p}\right] \ddot{\boldsymbol{\kappa}}=} & \frac{1}{2}\left[\frac{\partial\left[\mathbf{M}_{p}\right]}{\partial \boldsymbol{\kappa}} \dot{\boldsymbol{\kappa}}\right]^{T} \dot{\boldsymbol{\kappa}}-\left[\dot{\mathbf{M}}_{p}\right] \dot{\boldsymbol{\kappa}}+\mathbf{F}_{p}\left(\dot{\theta}_{m}, \ddot{\theta}_{m}\right) \\
& +\mathbf{Q}_{p}-\frac{\partial V_{p g}}{\partial \boldsymbol{\kappa}},
\end{aligned}
$$


TABle 1: Parameter setup.

\begin{tabular}{|c|c|c|c|}
\hline Parameters & Note & Nominal value & Unit \\
\hline Mass of the core assembly excluding the motor & $m_{a}$ & 62 & $\mathrm{~kg}$ \\
\hline Mass of the motor & $m_{m}$ & 18 & $\mathrm{~kg}$ \\
\hline Distance between axes of the motor and the core assembly & $D_{m}$ & 0.36 & $\mathrm{~m}$ \\
\hline Mass of the unbalance & $m_{u}$ & 2 & $\mathrm{~kg}$ \\
\hline Height of the unbalance relative to the suspension plane & $h_{u}$ & 0.1 & $\mathrm{~m}$ \\
\hline Gyration radius of the unbalance & $r_{u}$ & 0.1 & $\mathrm{~m}$ \\
\hline Stiffness of the suspension rods & $K_{1}$ & $7.78 \times 10^{7}$ & $\mathrm{~N} \mathrm{~m}^{-1}$ \\
\hline Stiffness of the compression springs & $K_{2}$ & 8000 & $\mathrm{Nm}^{-1}$ \\
\hline Pretightening force of the compression springs & $F_{\text {pre }}$ & 100 & $\mathrm{~N}$ \\
\hline Distance between two supporting legs & $D_{s}$ & 0.468 & $\mathrm{~m}$ \\
\hline Initial length of the rods & $l_{0}$ & 0.1 & $\mathrm{~m}$ \\
\hline Axial frictional damping force of the rods & $F_{f}$ & 10 & $\mathrm{~N}$ \\
\hline Coefficient of friction in the spherical joints & $\mu$ & 0.1 & - \\
\hline Radius of the spherical joints & $r$ & 0.02 & $\mathrm{~m}$ \\
\hline Gyration radius of the balancer & $r_{b}$ & 0.13 & $\mathrm{~m}$ \\
\hline Installing height of the balancer relative to the suspension plane & $h_{b}$ & 0.2 & $\mathrm{~m}$ \\
\hline Mass of each ball in the balancer & $m_{b}$ & 1 & $\mathrm{~kg}$ \\
\hline Viscous damping coefficient in the balancer & $c_{b}$ & 0.05 & $\mathrm{Nms} \mathrm{rad}{ }^{-1}$ \\
\hline Transmission ratio between the motor and the bowl & $i_{t}$ & 1 & - \\
\hline
\end{tabular}

where $\boldsymbol{\kappa}=\left[\begin{array}{llllllll}x & y & z & \alpha & \beta & \gamma & \phi_{1} & \phi_{2}\end{array}\right]^{T}$;

$$
\mathbf{Q}_{p}=\left[\begin{array}{c}
\mathbf{Q} \\
-c_{b} \dot{\phi}_{1} \\
-c_{b} \dot{\phi}_{2}
\end{array}\right]
$$

with $c_{b}$ representing the coefficient of viscous damping between the balls and the cavity and $\dot{\phi}_{i}$ denoting the rotational speed of the $i$ th ball relative to the bowl. The absolute rotational speed of the ball in $X_{b} Y_{b} Z_{b}$ can be expressed as $\dot{\phi}_{i}+\dot{\theta}$ where $\dot{\theta}$ is the spin speed of the bowl in $X_{b} Y_{b} Z_{b}$. A detailed illustration of (2) can be found in [8].

The generalized forces $\mathbf{Q}$ provided by the suspension system are a little different from those of the washing machine discussed in [7-11]. In the washing machine, the spring shown in Figure 1(b) is usually installed under the lower spherical joint. If the supporting bed moves lower along the rod, the spring will compress, which makes the suspension system very soft. However, in the centrifuge, the supporting bed is mainly suspended by the rod and the spring is only used for avoiding jumping of the supporting bed from the lower joint. Considering that the stiffness of the rod is much higher than that of the spring, the force acting on the supporting bed by the rod is estimated by

$$
F_{a} \approx \begin{cases}K_{2}\left(l-l_{0}\right)-F_{\text {pre }}-F_{f} \operatorname{sgn}(v) & l<l_{0} \\ K_{1}\left(l-l_{0}\right)-F_{\text {pre }}-F_{f} \operatorname{sgn}(v) & l \geq l_{0},\end{cases}
$$

where $l$ and $l_{0}$ denote the dynamic and initial distances between the upper joint and the bed, respectively; $F_{\text {pre }}$ is the pretightening force acting on the bed by the spring; $K_{1}$ and $K_{2}$ are the stiffness coefficients of the rod and the spring, respectively; $F_{f}$ is the frictional force between the bed and the rod; $v$ is the translational velocity of the bed relative to the rod.

Considering that the moments acting on the spherical joints are influenced by axial forces, frictional damping is assumed in all joints. The descriptions of the moments in [11] caused by viscous damping are transformed into frictional models. Take the moment acting on the upper joint shown in Figure 1(b) for example; due to frictional damping [12], it can be described as

$$
M_{p z}= \begin{cases}-\operatorname{sgn}(\omega) r \mu\left(F_{a}+F_{\mathrm{pre}}\right) & l>l_{0} \\ 0 & l \leq l_{0}\end{cases}
$$

where $l \leq l_{0}$ implies that the joint is detached; $r$ is the radius of the joint; $\mu$ denotes the frictional coefficient; for simplicity reason, the stick and slip frictional coefficients in all joints are assumed to be the same; $\omega$ is the rotational speed of the rod relative to the ground. The moments acting on other joints can also be transformed using the same way and no more derivations will be carried out here.

\section{Discussions}

This section explores dynamics of the three-column centrifuge with and without the ball balancer. The design parameters of the centrifuge including their notes, nominal values, and units are listed in Table 1.

\subsection{Dynamics of the Three-Column Centrifuge}

3.1.1. Modal Analysis. For a better understanding of the suspension characteristics, this section explores natural frequencies of the centrifuge itself. 


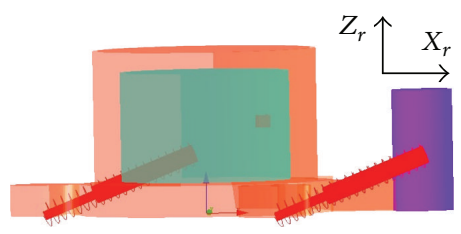

(a) The 1st order

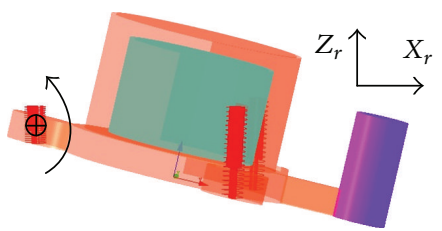

(d) The 4th order

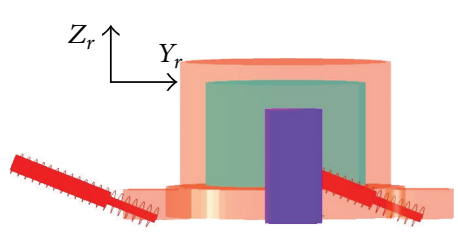

(b) The 2nd order

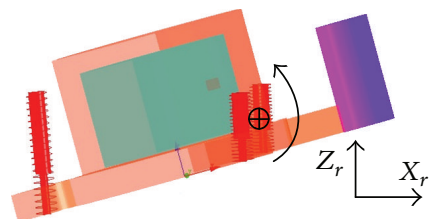

(e) The 5th order

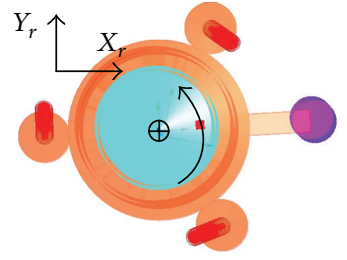

(c) The 3rd order

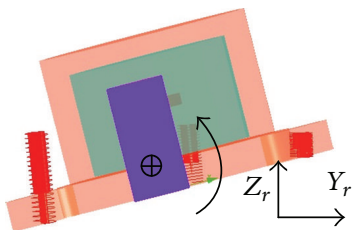

(f) The 6th order

FIgURE 2: Mode shapes of the system. “ $\oplus$ ” denotes the rotational center.

TABLE 2: Natural frequencies of the centrifuge.

\begin{tabular}{lcccccc}
\hline Order & 1st & 2nd & 3rd & 4th & 5th & 6th \\
\hline Frequency $(\mathrm{Hz})$ & 1.576 & 1.576 & 2.059 & 219.84 & 332.13 & 418.32 \\
\hline
\end{tabular}

The vibration model described in (1) is linearized at its static equilibrium position. Let the generalized velocities and accelerations in (1) be equal to zero; that is, $\dot{\mathbf{q}}=\ddot{\mathbf{q}}=\mathbf{0}$ and $\dot{\theta}_{m}=\ddot{\theta}_{m}=0$; the static equilibrium condition can be expressed as

$$
\mathbf{f}(\mathbf{q})=\mathbf{Q}-\frac{\partial V_{g}}{\partial \mathbf{q}}=\mathbf{0} .
$$

The static equilibrium position can be obtained by NewtonRaphson method

$$
\begin{aligned}
\mathbf{q}_{n+1}^{*} & =\mathbf{q}_{n}^{*}-\left.\frac{\partial \mathbf{f}(\mathbf{q})}{\partial \mathbf{q}}\right|_{\mathbf{q}=\mathbf{q}_{n}^{*}} ^{-1} \mathbf{f}\left(\mathbf{q}_{n}^{*}\right) \\
& =\mathbf{q}_{n}^{*}-\left[\mathbf{K}\left(\mathbf{q}_{n}^{*}\right)\right]^{-1} \mathbf{f}\left(\mathbf{q}_{n}^{*}\right),
\end{aligned}
$$

where $\mathbf{q}^{*}$ represents the static equilibrium position and $[\mathbf{K}(\mathbf{q})]=\partial \mathbf{f}(\mathbf{q}) / \partial \mathbf{q}$ is the Jacobian matrix. Natural frequencies can be obtained based on the following linearized system:

$$
\left[\mathbf{M}\left(\mathbf{q}^{*}\right)\right] \ddot{\mathbf{q}}+\left[\mathbf{K}\left(\mathbf{q}^{*}\right)\right] \mathbf{q}=\mathbf{0},
$$

where $\left[\mathbf{M}\left(\mathbf{q}^{*}\right)\right]$ and $\left[\mathbf{K}\left(\mathbf{q}^{*}\right)\right]$ are the mass and Jacobian matrices at $\mathbf{q}^{*}$, respectively.

Generalized eigenvalues and vectors can be solved from (8), and, during the process, nominal parameter values in Table 1 are employed. The natural frequencies are listed in Table 2 which are the square roots of the eigenvalues. As can be seen, the frequencies can be separated into two isolated groups: the first group comprises the first-, second, and third-order natural frequencies, while the other one is composed of the fourth-, fifth-, and sixth-order ones.

The first-, second-, and third-order mode shapes are displayed in Figures 2(a), 2(b), and 2(c), respectively. As
Figures 2(a) and 2(b) show, the three rods swing along $X_{r}$ - or $Y_{r}$-direction simultaneously and the centrifuge performs like a single pendulum. In fact, the first two natural frequencies can also be estimated by

$$
\omega_{n}=\sqrt{\frac{g}{l_{e}}}
$$

where $g$ is the acceleration of gravity and $l_{e}$ is the length of the rods at the equilibrium position which is very close to $l_{0}$. Assuming that $g=9.86 \mathrm{~m} \mathrm{~s}^{-2}$, the first two natural frequencies can be calculated as $\sqrt{9.86 / 0.1} /(2 \pi) \approx 1.58 \mathrm{~Hz}$. As can been seen, the result almost equals that listed in Table 2.

The third-order mode shape is shown in Figure 2(c) which behaves in a way that the core system rotates around $Z_{r}$-axis. As can be seen, no elongations or contractions of the rods occur in the first three mode shapes, which makes the corresponding natural frequencies so small.

The fourth-, fifth-, and sixth-order mode shapes are displayed in Figures 2(d), 2(e), and 2(f), respectively. It can be seen that the fourth- and fifth-order mode shapes are similar to each other: both of them rotate around an axis parallel to $Y_{r}$-axis. However, their rotational centers denoted by “ $\oplus$ " are different. Figure 2(e) displays the sixth-order mode shape; as the figure reveals, it rotates around $X_{r}$-axis. As can be seen, elongations or contractions of the rods are involved in the three figures. Considering that the rods are very stiff, the corresponding natural frequencies have to be big.

3.1.2. Dynamics of the Three-Column Centrifuge. This section discusses dynamics of the three-column centrifuge itself. Considering that the rods are very stiff, vibration angles of the centrifuge around $X_{r^{-}}$and $Y_{r}$-axes are quite small; this makes dynamics of the system very similar to that of a planar rotor. Once above the third-order natural frequency, at the steady state, the centrifuge will rotate around the equivalent mass center of the whole system. We now analyze centrifugal forces acting on the system at the steady state. In Figure 3, $J_{1}$, 


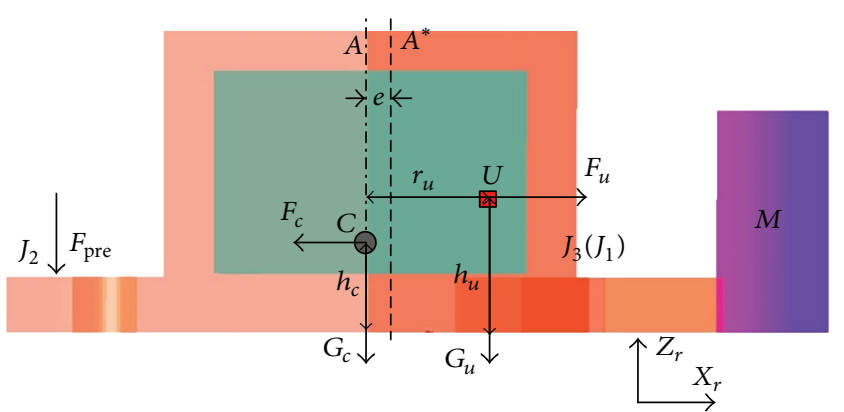

(a)

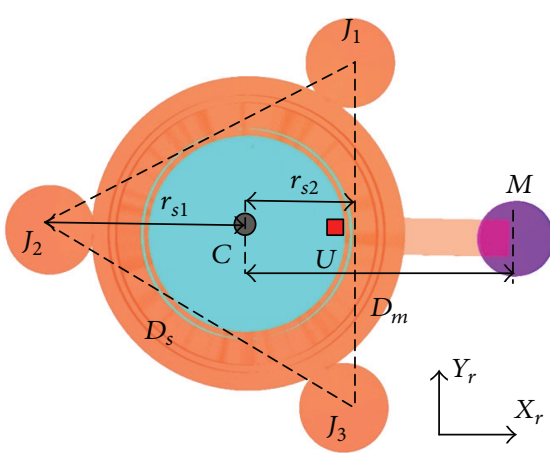

(b)

FIGURE 3: Forces acting on the system at the steady state when no balancer is considered: front view (a); top view (b).

$J_{2}$, and $J_{3}$ are the three hanging joints and $F_{\text {pre }}$ denotes the pretightening force applied on the joints by the spring shown in Figure 1(b). Assuming that the three joints are separated evenly along circumferential direction, distance between any two of them $D_{s}$ is identical. The distance between the central axis of the assembly $A$ and joint $J_{2}$ can be derived as $r_{s 1}=$ $D_{s} / \sqrt{3}$, and the distance between $A$ and $J_{1} J_{3}$ is $r_{s 2}=r_{s 1} / 2$.

The equivalent mass center of the core assembly including the motor is denoted by $C$ and the mass center of the unbalance is represented by $U$. For a general description, the asymmetry of the system caused by the motor $M$ is neglected and the equivalent mass center $C$ is assumed to be aligned with $A$. After the unbalance is involved, the equivalent mass center of the whole system will be aligned with $A^{*}$ around which the whole system will rotate at the steady state. The distance between $A$ and $A^{*}$ is the eccentricity of the whole system which can be calculated as

$$
e=\frac{m_{u} r_{u}}{\left(m_{u}+m_{c}\right)}
$$

where $m_{u}$ and $m_{c}$ denote mass of the unbalance and that of the core assembly including the motor, respectively; $r_{u}$ represents the gyration radius of the unbalance. The gravity forces acting on $C$ and $U$ are $G_{c}=m_{c} g$ and $G_{u}=m_{u} g$, respectively. And the centrifugal force acting on $C$ is

$$
F_{c}=m_{c} e \Omega^{2},
$$

where $\Omega$ denotes the operating speed. The centrifugal force acting on $U$ can be expressed as

$$
F_{u}=m_{u}\left(r_{u}-e\right) \Omega^{2} .
$$

At the steady state, the centrifugal forces $F_{c}$ and $F_{u}$ will counteract each other and an equilibrium between them will occur. Considering that, in a real centrifuge, the height of $U$ relative to the suspension plane is usually higher than that of $C$, moments generated by $F_{c}$ and $F_{u}$ cannot counteract each other completely, and the difference between them $F_{c} h_{c}-$ $F_{u} h_{u}$ has to be compensated by other moments such as those generated by the gravity. Considering that both $F_{c}$ and $F_{u}$ are proportional to $\Omega^{2}$, if $\Omega$ grows, the difference $F_{c} h_{c}-F_{u} h_{u}$ could be large enough to tip-over the core assembly. And that is the very reason why the centrifuge cannot run very high. We now discuss the critical speed above which the centrifuge will tip-over. The smallest tip-over speed appears in Figure 3 where all the moments relative to $J_{1} J_{3}$ are in a critical equilibrium condition

$$
\begin{aligned}
& F_{u} h_{u}-F_{c} h_{c}-G_{c} r_{s 2}-G_{u}\left(r_{s 2}-r_{u}\right)-F_{\mathrm{pre}}\left(r_{s 1}+r_{s 2}\right) \\
& \quad=0
\end{aligned}
$$

where $r_{s 2}=(\sqrt{3} / 6) D_{s}$. Submitting (11), (12), $G_{u}=m_{u} g$, and $G_{c}=m_{c} g$ into (13) leads to

$$
\Omega_{1}^{*}=\sqrt{\frac{m_{c} g r_{s 2}+m_{u} g\left(r_{s 2}-r_{u}\right)+3 F_{\mathrm{pre}} r_{s 2}}{m_{u}\left(r_{u}-e\right) h_{u}-m_{c} e h_{c}}} \quad h_{c}<h_{u} .
$$

If $\Omega>\Omega_{1}^{*}$, tip-over phenomenon of the assembly relative to $J_{1} J_{3}$ will occur and $J_{2}$ will detach. This could cause serous vibrations of the system, especially when a small axial damping of the rods, such as the one shown in Figure 1(b), is employed. For a general description of the tip-over speed, the asymmetry of the system caused by the motor was not considered during the above derivations, and the result calculated by (14) may be a little higher than the real one. This can be seen from Figure 3 where, at this very moment, the gravity force acting on the motor makes the tip-over phenomenon more easily occur. Equation (14) can be adjusted by taking the gravity moment generated by the motor into consideration

$$
\begin{array}{r}
\Omega_{1}^{*} \\
\frac{\frac{m_{c} g r_{s 2}+m_{u} g\left(r_{s 2}-r_{u}\right)+3 F_{\mathrm{pre}} r_{s 2}-m_{m} g\left(D_{m}-r_{s 2}\right)}{m_{u}\left(r_{u}-e\right) h_{u}-m_{c} e h_{c}}}{h_{c}<h_{u},}
\end{array}
$$

where $m_{c}=m_{a}+m_{m}$ with $m_{a}$ and $m_{m}$ denoting mass of the assembly excluding the motor and that of the motor itself, respectively; $D_{m}$ denotes the distance from the mass center of the motor to the central axis $A$. 


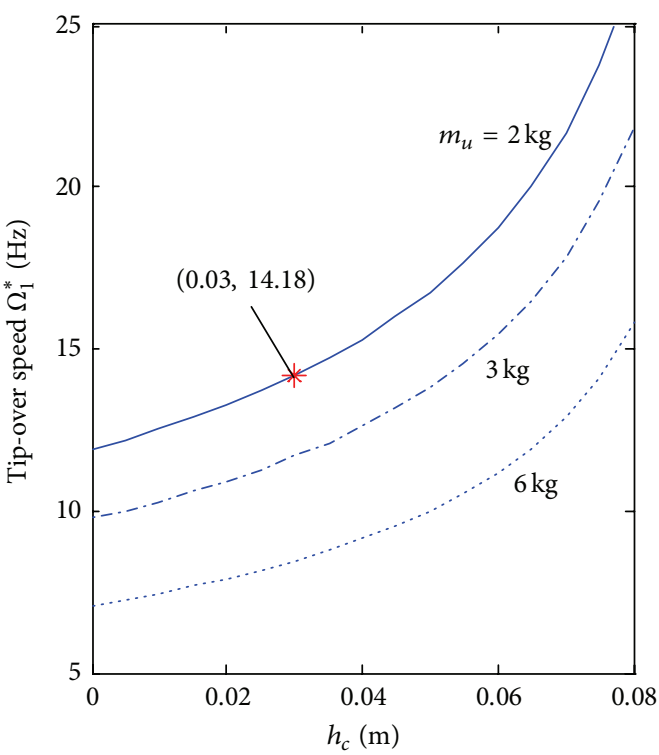

(a)

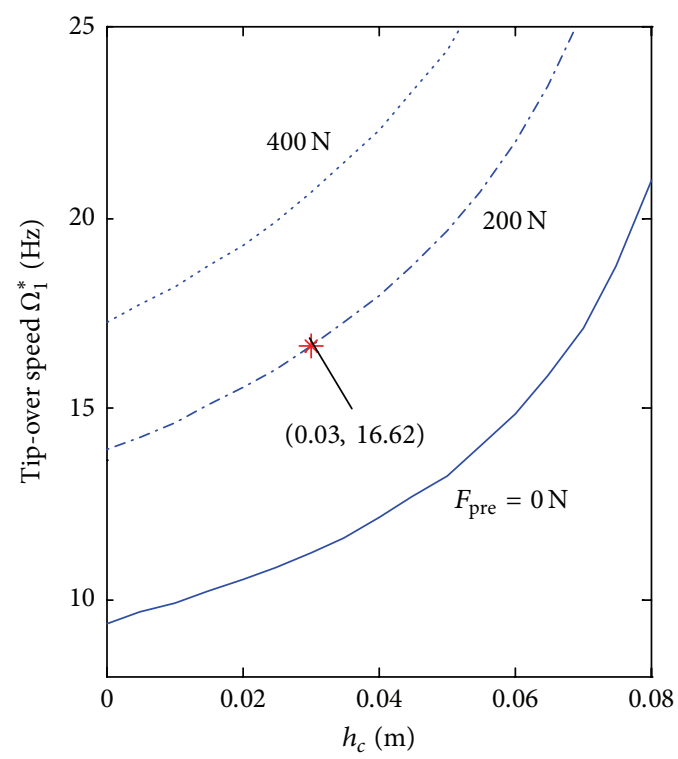

(b)

Figure 4: Variations of the tip-over speed $\Omega_{1}^{*}$ along with increases of $m_{u}$ and $h_{c}$ when pretightening force $F_{\text {pre }}=100 \mathrm{~N}$ (a). Changes of the tip-over speed $\Omega_{1}^{*}$ along with variations of $F_{\text {pre }}$ and $h_{c}$ when $m_{u}=2 \mathrm{~kg}(\mathrm{~b})$.

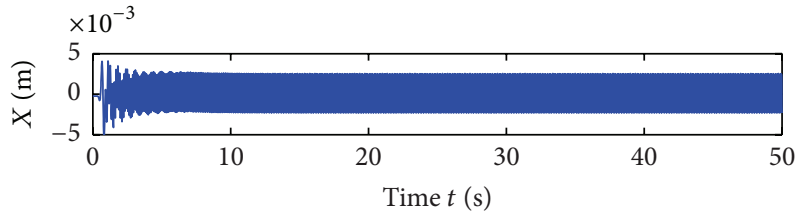

(a)

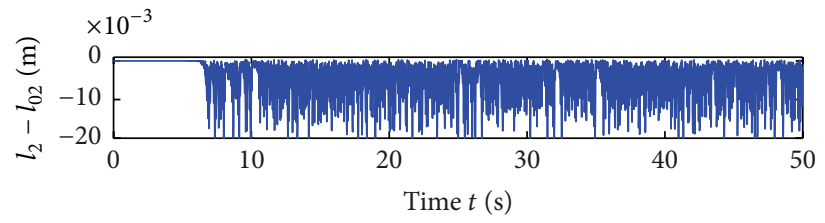

(c)

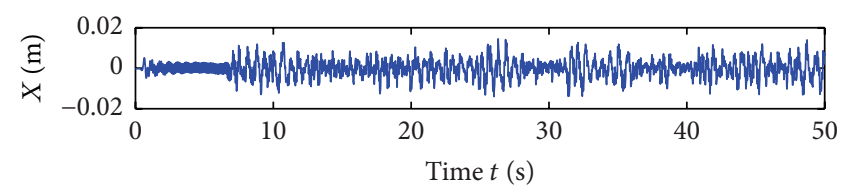

(b)

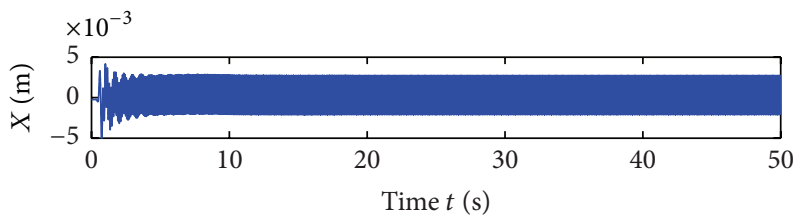

(d)

Figure 5: $X$ displacement of the core assembly when $\Omega=13.5 \mathrm{~Hz}, F_{\text {pre }}=100 \mathrm{~N}(\mathrm{a}) ; \Omega=14.5 \mathrm{~Hz}, F_{\text {pre }}=100 \mathrm{~N}$ (b); elongations of the second suspension $\operatorname{rod}$ when $\Omega=14.5 \mathrm{~Hz}, F_{\text {pre }}=100 \mathrm{~N}(\mathrm{c})$; and $X$ displacement of the core assembly when $\Omega=16 \mathrm{~Hz}, F_{\text {pre }}=200 \mathrm{~N}(\mathrm{~d})$.

Figure 4 displays variations of the tip-over speed $\Omega_{1}^{*}$ along with increases of $h_{c}, m_{u}$, and $F_{\text {pre }}$ when height of the unbalance relative to the suspension plane $h_{u}=0.1 \mathrm{~m}$. As can be seen from Figure 4(a), in the interval $[0,0.1)$, the tip-over speed $\Omega_{1}^{*}$ grows larger while $h_{c}$ increases. Different types of unbalance $m_{u}$ are considered; as the figure reveals, a bigger $m_{u}$ corresponds to a smaller $\Omega_{1}^{*}$. Figure 4(b) shows variations of $\Omega_{1}^{*}$ along with the increase of $h_{c}$ when $m_{u}=2 \mathrm{~kg}$. Different pretightening forces $F_{\text {pre }}$ are considered. As can be seen, a bigger $\Omega_{1}^{*}$ can be obtained by a stronger $F_{\text {pre }}$.

We now validate the above results. Simulations are carried out using Runge-Kutta fourth-order method based on (1). During the process, nominal parameter values in Table 1 are used and the operating speed of the centrifuge is assumed to be

$$
\dot{\theta}=\Omega\left(1-\mathrm{e}^{-t / \tau}\right),
$$

where $\Omega$ is the operating speed at the steady state and $\tau$ is a time constant. In this paper, the value $\tau=1$ is adopted.

Figure 5 shows $X$ displacement of the core assembly under different conditions. As Figure 4(a) reveals, the tipover speed $\Omega_{1}^{*}$ is $14.18 \mathrm{~Hz}$ when $F_{\text {pre }}=100 \mathrm{~N}$ and $m_{u}=2 \mathrm{~kg}$. Time responses corresponding to $\Omega=13.5 \mathrm{~Hz}$ and $\Omega=$ $14.5 \mathrm{~Hz}$ are displayed in Figures 5(a) and 5(b), respectively. As can be seen, a stable periodical solution is achieved in Figure 5(a) while an unstable one is obtained in Figure 5(b). 


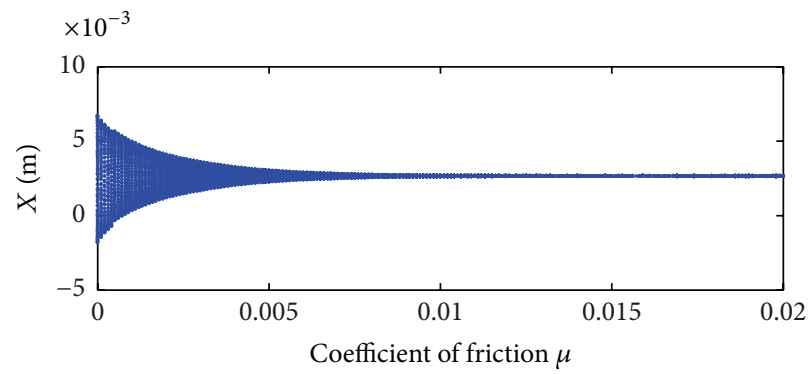

(a)

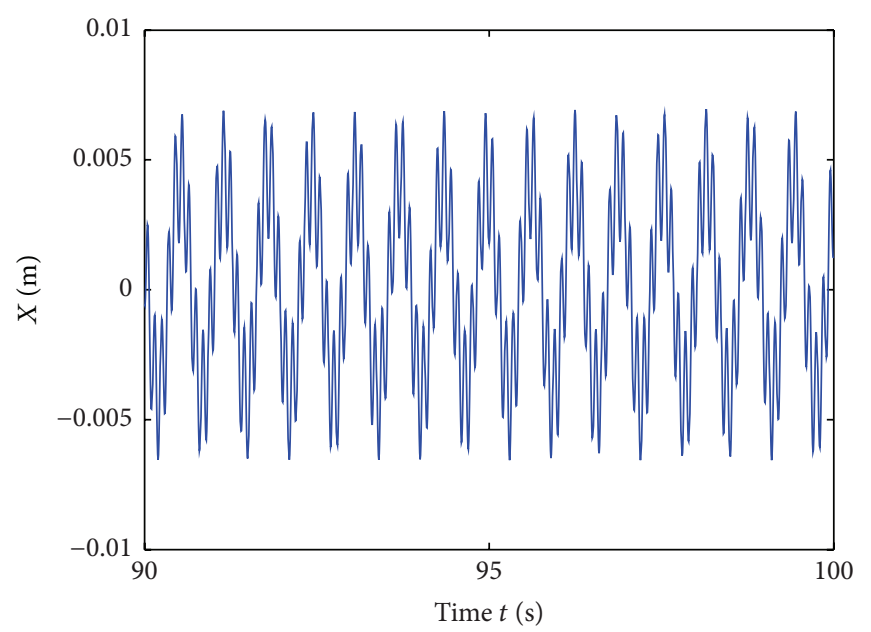

(b)

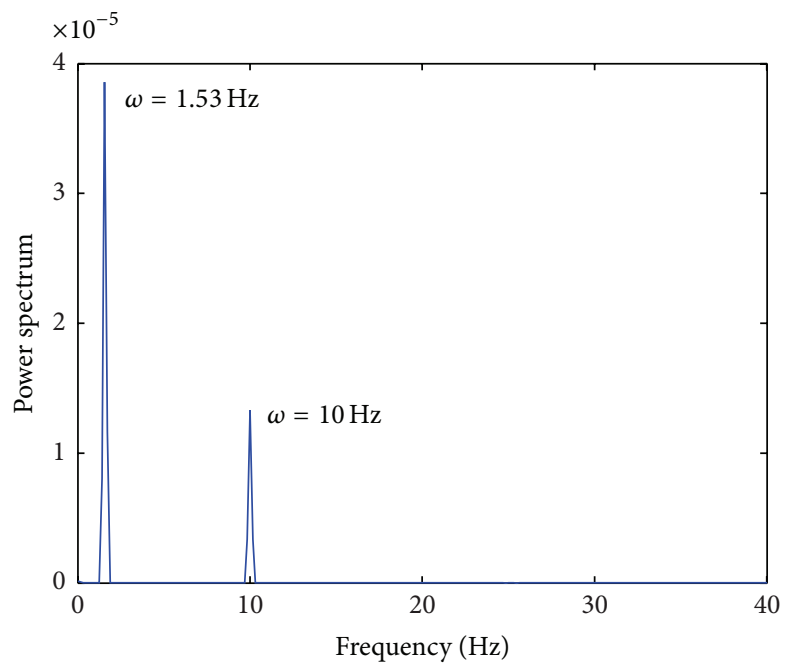

(c)

FIgURE 6: Bifurcation diagram of the system as $\mu$ varies (a); $X$ displacement when $\mu=0$ (b); Power spectrum (c).

Figure 5(c) displays elongations of the second suspension rod when $\Omega=14.5 \mathrm{~Hz}$. As the figure reveals, $l_{2}$ is sometimes smaller than $l_{02}$ in the interval $(6,50]$ which implies that the spherical joint $J_{2}$ is sometimes detached. Serious vibrations can be seen from Figure 5(b) in the interval $(6,50]$. For alleviating the situation, a larger pretightening force can be adopted. According to Figure 4(b), a pretightening force equal to $200 \mathrm{~N}$ can guarantee a stable solution even after the operating speed increases to $\Omega=16 \mathrm{~Hz}$. The corresponding time response is shown in Figure 5(d); as can be seen, a stale solution is obtained.

We now discuss influences of the frictional damping in the spherical joints on dynamics of the system. Figure $6(\mathrm{a})$ shows bifurcation diagram of the system along with the variation of the frictional coefficient $\mu$ when $\Omega=10 \mathrm{~Hz}, m_{u}=$ $2 \mathrm{~kg}, r_{u}=0.1 \mathrm{~m}, h_{u}=0.1 \mathrm{~m}, h_{c}=0.03 \mathrm{~m}$, and $F_{\text {pre }}=0 \mathrm{~N}$. As can be seen, instability happens when $\mu$ decreases. The time response and power spectrum of $X$ displacement when $\mu=0$ are shown in Figures 6(b) and 6(c), respectively. Two isolated peaks can be identified in Figure 6(c). The one at $\omega=10 \mathrm{~Hz}$ corresponds to the operating speed of the centrifuge, and the other one at $\omega=1.563 \mathrm{~Hz}$ is very close to the firstorder (or second-order) natural frequency of the system. This phenomenon is very similar to the instability that happens in a vertical axis washing machine studied in our previous paper [11] which is mainly caused by a Hopf bifurcation due to small damping in the spherical joints.

Simulations reveal that, for a three-column centrifuge, once the operating speed is higher than $20 \mathrm{~Hz}$, in order to suppress the instability, both the frictional coefficient $\mu$ and the axial damping of the rods $c_{a}$ have to be big. Figure 7 displays $X$ displacement and the corresponding power spectrum when $\Omega=24 \mathrm{~Hz}, h_{c}=0.1 \mathrm{~m}, F_{\text {pre }}=100 \mathrm{~N}$, $c_{a}=200 \mathrm{~N}$, and $\mu=2$. As can be seen, even though a large axial damping $c_{a}$ and a big $\mu$ are applied, the instability can still not be completely eliminated. According to (15), if $h_{c}$ equals $h_{u}=0.1 \mathrm{~m}, \Omega_{1}^{*}$ should be infinite; however, due to the instability born with the suspension structure, the maximum operating speed of the centrifuge itself might be constrained. 


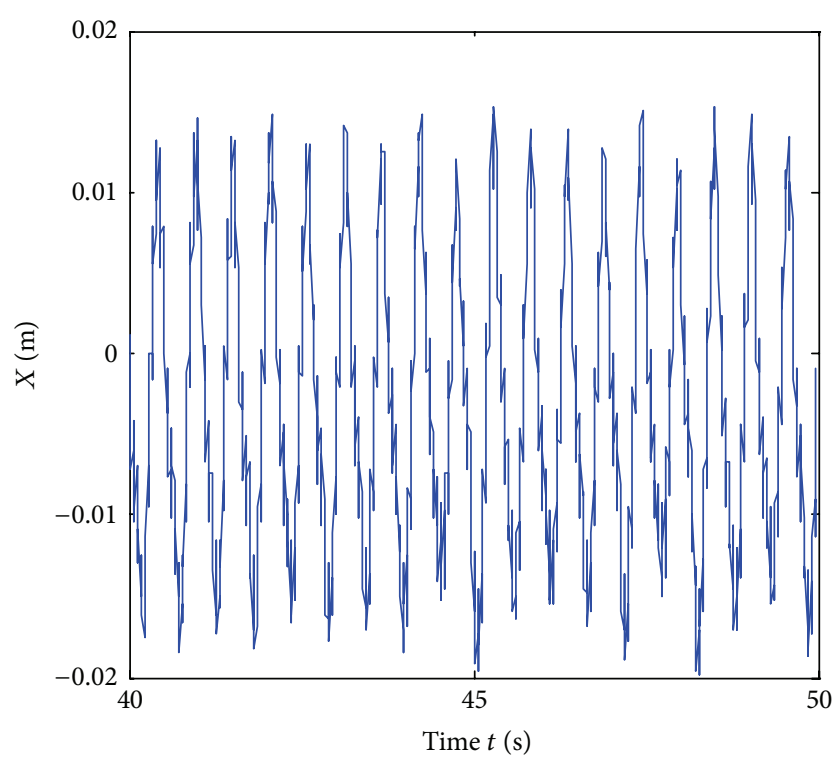

(a)

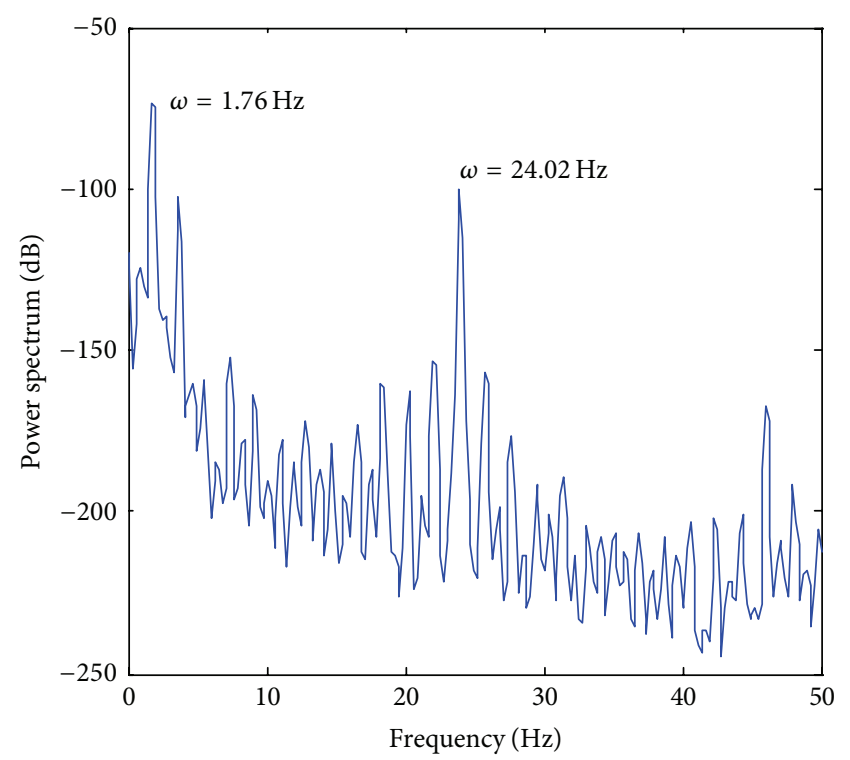

(b)

FIgURE 7: $X$ displacement when $\Omega=24 \mathrm{~Hz}, h_{c}=0.1 \mathrm{~m}, F_{\text {pre }}=100 \mathrm{~N}, c_{a}=200 \mathrm{~N}$ and $\mu=2$ (a); power spectrum (b).

3.2. Dynamics of the Three-Column Centrifuge with a Ball Balancer. This section studies dynamics of the three-column centrifuge when a ball balancer is employed. A two-ball model is employed and no collision happening between the balls is assumed. Besides, for exploring fundamental dynamics of the system, only viscous damping is assumed in the balancer.

Instability due to the balancer usually happens in the neighborhood of the critical frequencies [13-15] of a rotor. Simulations reveal that instability of the balancer does occur near the first-order (or second-order) natural frequency of the three-column centrifuge; however, the influence can be neglected because the operating speed when instability happens is only about $2 \mathrm{~Hz}$; at this circumstance, vibrations caused by the instability are too small to be considered. Thus, instability characteristics of the balancer will not be presented in this paper.

The study in this section mainly focuses on the tipover phenomenon of the three-column centrifuge after the balancer is employed. This phenomenon usually occurs at the steady state when the operating speed is much higher than the first-order (or second-order) natural frequency of the system. During the studies, no rolling friction and no collisions between the balls are assumed in the balancer and both of the two balls could reach their optimal positions.

Centrifugal forces acting on the system at the steady state are shown in Figure 8. As Figure 8(b) displays, the dashed circle $B$ represents the equivalent mass center of the two balls Ball 1 and Ball 2. Forces acting on $B$ are the centrifugal force $F_{b}=2 m_{b} r_{b e} \Omega^{2}$ and the gravity force $G_{b}=2 m_{b} g$ where $r_{b e}$ is the equivalent gyration radius of $B$. If mass of the balls is small, at the steady state, the resultant centrifugal force $F_{b}$ is not enough to counteract $F_{u}$, and, in this situation, equilibrium of the centrifugal forces is governed by the following equation:

$$
F_{u}-F_{b}-F_{c}=0,
$$

where $F_{b}=2 m_{b}\left(r_{b e}+e_{2}\right) \Omega^{2}, F_{u}=m_{u}\left(r_{u}-e_{2}\right) \Omega^{2}$, and $F_{c}=$ $m_{c} e_{2} \Omega^{2}$, from which the eccentricity of the system $e_{2}$ can be derived as

$$
e_{2}=\frac{\left(m_{u} r_{u}-2 m_{b} r_{b e}\right)}{\left(m_{u}+2 m_{b}+m_{c}\right)}
$$

Figure 9 displays variations of $r_{b e}, F_{c}, e_{2}, F_{u}-F_{c}-F_{b}$, and $2 m_{b} r_{b e}$ along with the increase of $m_{b}$ when $\Omega=8 \mathrm{~Hz}$ and $h_{c}=0.03 \mathrm{~m}$. As can be seen from Figure 9(a), when mass of the balls $m_{b}$ is small, the two-ball group together at the opposite side of the unbalance and $r_{b e}$ equals $r_{b}=0.13 \mathrm{~m}$. However, once $m_{b}$ grows bigger than $0.8 \mathrm{~kg}, r_{b e}$ gets smaller and smaller. Figure $9(\mathrm{~b})$ reveals changes of the centrifugal force acting on $C$ when $m_{b}$ varies; as can be seen, at first, $F_{c}$ decreases as $m_{b}$ increases; however, once $m_{b}$ is beyond $0.8 \mathrm{~kg}$, $F_{c}$ almost turns to zero; in this situation, the eccentricity of the system shown in Figure 9(c) almost disappears. Figure 9(d) shows variations of the resultant force $F_{u}-F_{b}-F_{c}$. As can be seen, the resultant force is very small, which proves the rationality of (17).

According to Figure 9, if mass of the balls $m_{b}$ is big enough, vibrations of the system will vanish and the centrifugal force $F_{c}$ will be zero. In this situation, (17) can be rewritten as

$$
F_{u}-F_{b}=0
$$




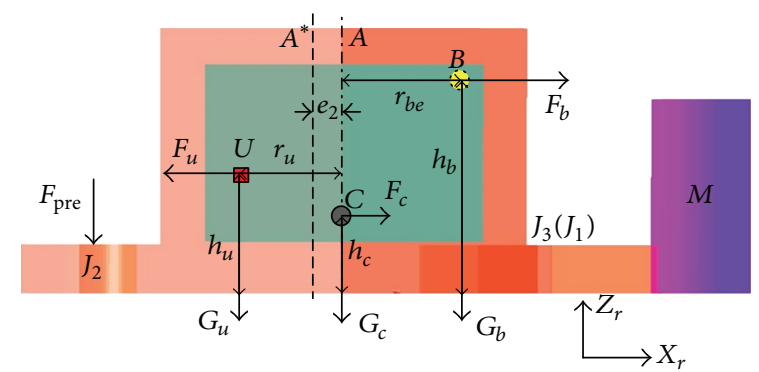

(a)

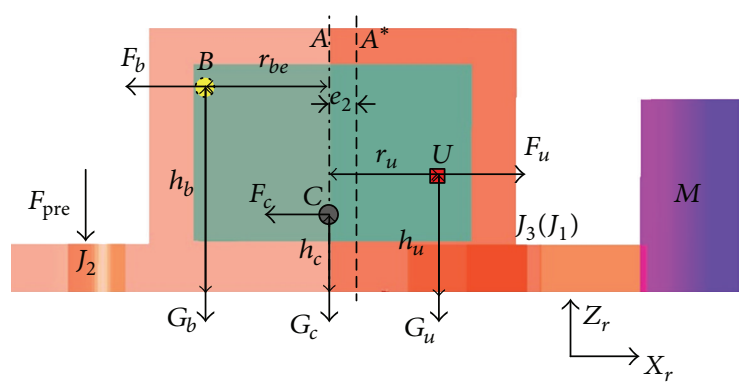

(c)

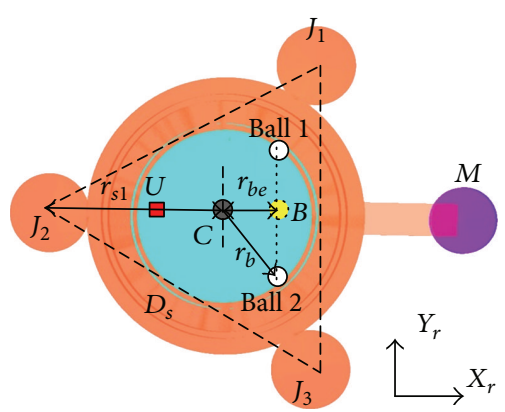

(b)

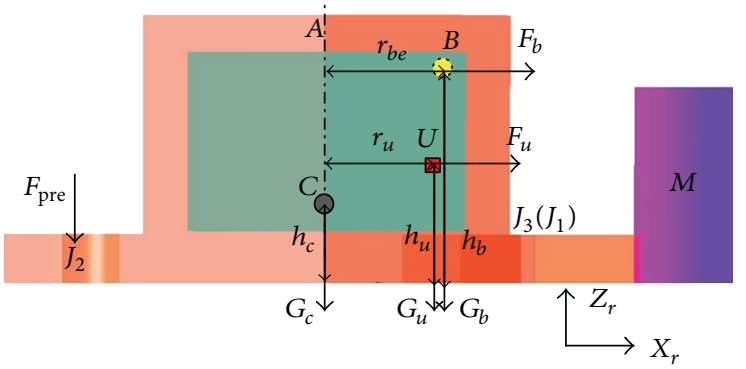

(d)

FIGURE 8: Forces acting on the system at the steady state when a ball balancer is considered.

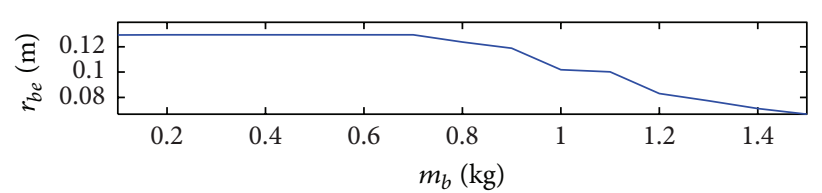

(a)

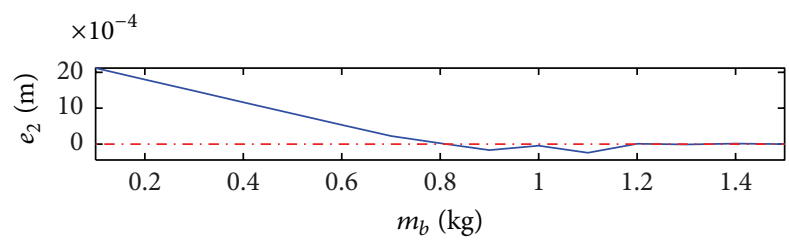

(c)

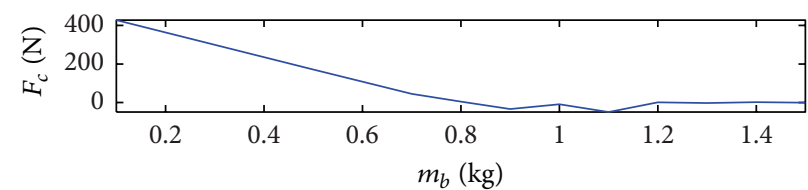

(b)

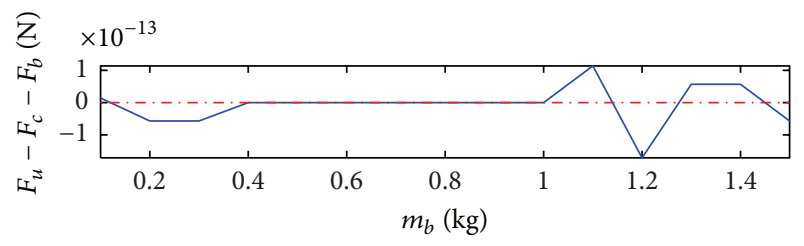

(d)

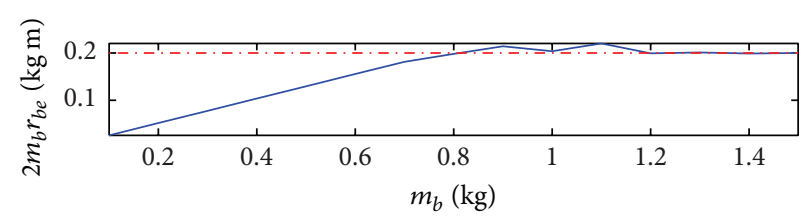

(e)

FiguRE 9: Variations of $r_{b e}, F_{c}, e_{2}, F_{u}-F_{c}-F_{b}$, and $2 m_{b} r_{b e}$ along with the increase of $m_{b}$ when $\Omega=8 \mathrm{~Hz}$ and $h_{c}=0.03 \mathrm{~m}$.

from which the critical value of $m_{b}$ can be derived after submitting $r_{b e}=r_{b}$ into the above equation

$$
m_{b}^{*}=\frac{m_{u} r_{u}}{2 r_{b}}=\frac{2 \times 0.1}{2 \times 0.13} \approx 0.77 \mathrm{~kg} .
$$

Actually, $m_{b}^{*}$ corresponds to the pitch-fork bifurcation point discussed in a planar rotor [15]. As Figure 9(e) reveals, once $m_{b}>m_{b 1}^{*}$, the product $2 m_{b} r_{b e}$ stays at a relatively stable value that equals $m_{u} r_{u}=0.2 \mathrm{~kg} \mathrm{~m}$; this is caused by the idea that the two balls are separated from each other and the gyration radius $r_{b e}$ decreases as $m_{b}$ increases in order to keep the equilibrium between $F_{u}$ and $F_{b}$.

Based on the above discussions, it can be seen that when mass of the balls $m_{b}$ is smaller than $m_{b}^{*}$, the two balls stick together and the equivalent gyration radius of the balls $r_{b e}$ equals $r_{b}$; however, once $m_{b} \geq m_{b}^{*}$, the equilibrium between $F_{u}$ and $F_{b}$ will appear which leads to $m_{u} r_{u}=2 m_{b} r_{b e}$. Thus, the 
equivalent gyration radius of the balls $r_{b e}$ can be summarized as

$$
r_{b e}= \begin{cases}r_{b} & m_{b}<m_{b}^{*} \\ \frac{m_{u} r_{u}}{2 m_{b}} & m_{b} \geq m_{b}^{*} .\end{cases}
$$

Although an equilibrium between $F_{u}$ and $F_{b}$ can be obtained once $m_{b} \geq m_{b}^{*}$, the tip-over phenomenon can still happen. As Figure 8(a) reveals, if the height of the balancer relative to the suspension system $h_{b}$ does not equal that of the unbalance $h_{u}$, a resultant moment relative to $J_{1} J_{3}$ will occur, which could lead to a tip-over phenomenon at a high operating speed. Actually, three different types of tipover phenomena may be induced; the first one is shown in Figure 8(a) which is caused by $F_{b} h_{b}+F_{c} h_{c}>F_{u} h_{u}$ and governed by

$$
\begin{gathered}
F_{b} h_{b}+F_{c} h_{c}-F_{u} h_{u}-G_{c} r_{s 2}-G_{b}\left(r_{s 2}-r_{b e}\right) \\
-G_{u}\left(r_{s 2}+r_{u}\right)-F_{\text {pre }}\left(r_{s 1}+r_{s 2}\right)=0 .
\end{gathered}
$$

The second type happens when $F_{b} h_{b}+F_{c} h_{c}<F_{u} h_{u}$; as shown in Figure 8(c), this situation is similar to that shown in Figure 3 and governed by

$$
\begin{gathered}
F_{u} h_{u}-F_{b} h_{b}-F_{c} h_{c}-G_{c} r_{s 2}-G_{b}\left(r_{s 2}+r_{b e}\right) \\
-G_{u}\left(r_{s 2}-r_{u}\right)-F_{\text {pre }}\left(r_{s 1}+r_{s 2}\right)=0 .
\end{gathered}
$$

A comparison can be made here between (23) and (13), it can be seen that two more items $-F_{b} h_{b}$ and $-G_{b}\left(r_{s 2}+r_{b e}\right)$ are counteracting $F_{u} h_{u}$ in (23) which is beneficial for obtaining a higher tip-over speed. It should be noted that, due to the pitch-fork bifurcation discussed in (19) and (20), $F_{b}$ is always smaller than or equal to $F_{u}$; if the height of the balancer $h_{b}$ is lower than $h_{u}$, no matter how big the mass of the balls $m_{b}$ is, the moment generated by $F_{b}$ is always smaller than that by $F_{u}$ and thus a tip-over phenomenon will always happen, even if the eccentricity of the system could reach zero.

We now discuss the third type of tip-over phenomenon that might occur at the transient stage. For doing this, the most serious condition is considered in Figure $8(\mathrm{~d})$. As can be seen, the unbalance and the balls are on the same side; at this circumstance, the equilibrium of the centrifugal moments can be estimated by

$$
\begin{aligned}
F_{u} h_{u} & +F_{b} h_{b}-G_{c} r_{s 2}-G_{b}\left(r_{s 2}-r_{b e}\right)-G_{u}\left(r_{s 2}-r_{u}\right) \\
& -F_{\text {pre }}\left(r_{s 1}+r_{s 2}\right)=0 .
\end{aligned}
$$

We now derive the tip-over speed. For a general description, asymmetry of the system caused by the motor is not considered in the above equations; this can be adjusted by taking the gravity moment generated by the motor into consideration. Different tip-over speeds can be derived based on the above equations and the one governed by (22) can be combined together with that governed by (23):

$$
\Omega_{2}^{*}= \begin{cases}\sqrt{\frac{2 m_{b} g\left(r_{s 2}+r_{b e}\right)+m_{c} g r_{s 2}+m_{u} g\left(r_{s 2}-r_{u}\right)+F_{\mathrm{pre}}\left(r_{s 1}+r_{s 2}\right)-m_{m} g\left(D_{m}-r_{s 2}\right)}{-\Delta}} & \Delta<0 \\ \sqrt{\frac{2 m_{b} g\left(r_{s 2}-r_{b e}\right)+m_{c} g r_{s 2}+m_{u} g\left(r_{s 2}+r_{u}\right)+F_{\mathrm{pre}}\left(r_{s 1}+r_{s 2}\right)-m_{m} g\left(D_{m}-r_{s 2}\right)}{\Delta}} & \Delta>0,\end{cases}
$$

where

$$
\Delta=2 m_{b}\left(r_{b e}+e_{2}\right) h_{b}+m_{c} e_{2} h_{c}-m_{u}\left(r_{u}-e_{2}\right) h_{u}
$$

and the item $m_{m} g\left(D_{m}-r_{s 2}\right)$ is employed for adjusting asymmetry of the system due to the motor. The value of $\Delta$ in (26) represents the status of the centrifugal moments. A positive $\Delta$ implies that $F_{b} h_{b}+F_{c} h_{c}>F_{u} h_{u}$, while a negative one indicates that $F_{b} h_{b}+F_{c} h_{c}<F_{u} h_{u}$. A zero $\Delta$ implies that equilibrium among the centrifugal moments has been achieved, and, at this circumstance, an infinite $\Omega_{2}^{*}$ can be obtained.

The tip-over speed governed by (24) can be derived as

$$
\Omega_{3}^{*}=\sqrt{\frac{m_{c} g r_{s 2}+2 m_{b} g\left(r_{s 2}-r_{b e}\right)+m_{u} g\left(r_{s 2}-r_{u}\right)+F_{\text {pre }}\left(r_{s 1}+r_{s 2}\right)-m_{m} g\left(D_{m}-r_{s 2}\right)}{m_{u} r_{u} h_{u}+2 m_{b} r_{b e} h_{b}}} .
$$

Considering that, at the transient stage, the equivalent gyration radius of the balls $r_{b e}$ is hard to calculate, the most serious condition $r_{b e}=r_{b}$ is assumed in (27).

Figure 10(a) displays variations of $\Omega_{2}^{*}$ and $\Omega_{3}^{*}$ along with the increase of $m_{b}$ when $m_{u}=2 \mathrm{~kg}, h_{b}=0.2 \mathrm{~m}, h_{u}=$
$0.1 \mathrm{~m}, h_{c}=0.03 \mathrm{~m}$, and $F_{\text {pre }}=100 \mathrm{~N}$. As can be seen, $\Omega_{3}^{*}$ decreases when $m_{b}$ grows bigger. This implies that, in order to avoid the tip-over phenomenon at the transient stage, a slower starting speed should be considered when a big balancer is employed. As the figure shows, in the interval $m_{b} \in[0,0.31)$, 


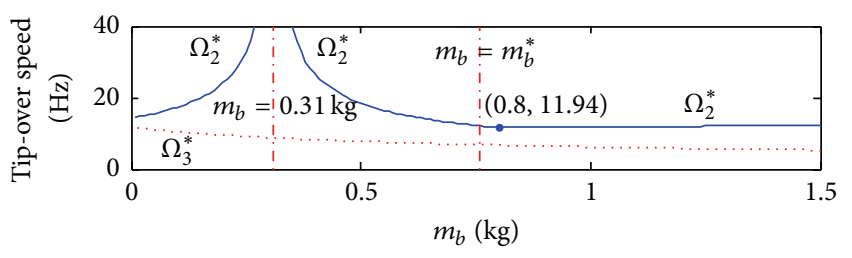

(a)

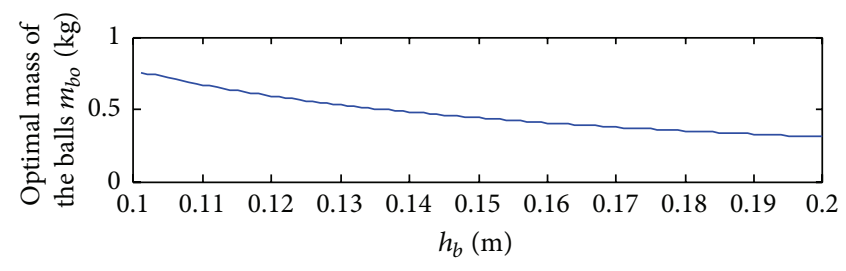

(b)

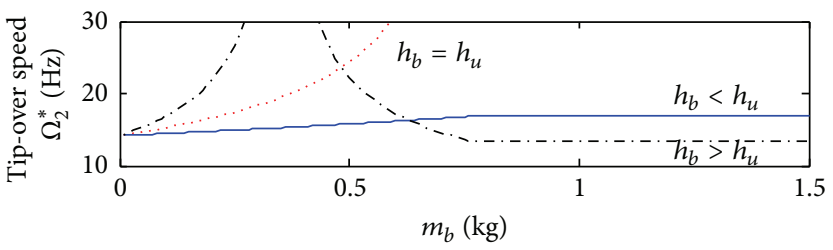

(c)

FIGURE 10: Variations of $\Omega_{2}^{*}$ and $\Omega_{3}^{*}$ along with increase of $m_{b}$ when $h_{b}=0.2 \mathrm{~m}$ (a). Variations of the optimal mass of the balls $m_{b o}$ with $h_{b}$ (b). Changes of $\Omega_{2}^{*}$ along with increase of $m_{b}$ when $h_{b}=0.2 \mathrm{~m}, h_{b}=h_{u}=0.1 \mathrm{~m}$, and $h_{b}=0.05 \mathrm{~m}$. Other parameter values are $m_{u}=2 \mathrm{~kg}$, $h_{u}=0.1 \mathrm{~m}, h_{c}=0.03 \mathrm{~m}$, and $F_{\text {pre }}=100 \mathrm{~N}$.

$\Omega_{2}^{*}$ increases quickly with $m_{b}$; however, once $m_{b}>0.31 \mathrm{~kg}$, $\Omega_{2}^{*}$ decreases rapidly in the interval $m_{b} \in\left(0.31, m_{b}^{*}\right]$ and then changes slowly once $m_{b}$ is bigger than $m_{b}^{*}$.

As Figure 10(a) reveals, when $h_{b}>h_{u}$, an optimal mass of the balls $m_{b}$ exists which could guarantee a very big $\Omega_{2}^{*}$. Actually, this optimal mass can be obtained by letting (26) be equal to zero; namely, $\Delta=0$. However, calculating $m_{b}$ directly from $\Delta=0$ is not easy, because the eccentricity $e_{2}$ is a function of $m_{b}$. This problem can be solved by numerical algorithms such as the bisection method. Figure 10(b) displays variations of the optimal mass of the balls $m_{b o}$ along with the increase of $h_{b}$. Considering that $h_{u}=0.1 \mathrm{~m}$, a bigger $m_{b o}$ can be obtained by a closer distance between $h_{b}$ and $h_{u}$, which is helpful for getting a smaller eccentricity of the system.

Figure 10(c) shows variations of $\Omega_{2}^{*}$ when different installing heights of the balancer $h_{b}$ are considered: $h_{b}=$ $0.2 \mathrm{~m}, h_{b}=h_{u}=0.1 \mathrm{~m}$, and $h_{b}=0.05 \mathrm{~m}$. As can be seen, if $h_{b}<h_{u}$, no matter how the balancer is designed, the tip-over speed $\Omega_{2}^{*}$ is always limited; if $h_{b}>h_{u}$, an optimal mass $m_{b o}$ exists; and if $h_{b}=h_{u}$, as long as $m_{b} \geq m_{b}^{*}$, complete balance of the centrifugal moments can be obtained which could lead to an infinite $\Omega_{2}^{*}$.

We now validate the conclusions made in Figure 10. Simulations are carried out using Runge-Kutta fourth-order method. The viscous damping coefficient in the balancer $c_{b}=$ $0.1 \mathrm{~N} \mathrm{~m} \mathrm{~s} \mathrm{rad}^{-1}$ is adopted and the initial positions of the balls are $\phi_{1}=0, \phi_{2}=\pi$.

Figures 11(a) and 11(b) display time responses when $h_{b}=$ $0.2 \mathrm{~m}$ and $m_{b}=0.8 \mathrm{~kg}$. According to Figure 10(a), the corresponding tip-over speed should be $\Omega_{2}^{*}=11.94 \mathrm{~Hz}$. Figures 11(a) and 11(b) display simulation results when $\Omega=$ $11.5 \mathrm{~Hz}$ and $\Omega=12.5 \mathrm{~Hz}$, respectively. As can be seen, the $X$ displacement in Figure 11(a) almost turns to zero at the steady state, while, in Figure 11(b), serious vibrations caused by the tip-over of the centrifuge can be observed.

Figure 11(c) displays variations of the positions of the first ball relative to the bowl when $h_{b}=0.2 \mathrm{~m}, \Omega=10 \mathrm{~Hz}$, and $m_{b}=1.5 \mathrm{~kg}$. As can be seen, when mass of the balls is much bigger than $m_{b}^{*}$, although the tip-over phenomenon does not occur, oscillations of the balls appear. This could make the tip-over speed $\Omega_{2}^{*}$ a little smaller than the theoretical one because $2 m_{b} r_{b e}$ may be sometimes bigger than $m_{u} r_{u}$ during the oscillations of the balls. Thus, a too big balancer is not recommended for the centrifuge.

Figure 11(d) displays the result when $m_{b}=0.31 \mathrm{~kg}, \Omega=$ $30 \mathrm{~Hz}$, and $\mu=0.3$. As can be seen, the tip-over speed $\Omega_{2}^{*}$ is much bigger than that in Figure 11(b) where $m_{b} \geq m_{b}^{*}$ is considered. However, due to the instability caused by a small $\mu$ shown in Figure 7, a big frictional coefficient in the spherical joints $\mu$ has to be applied.

Figure 11(e) shows the simulation result when $h_{b}=0.1 \mathrm{~m}$, $m_{b}=1.5 \mathrm{~kg}$, and $\Omega=100 \mathrm{~Hz}$. For avoiding the tip-over phenomenon during the transient stage, the starting speed of the system $\Omega_{\text {st }}$ is $7 \mathrm{~Hz}$ which is smaller than $\Omega_{3}^{*}$; then the speed is increased slowly from $7 \mathrm{~Hz}$ to $100 \mathrm{~Hz}$ with a step of $\Delta \Omega=2 \mathrm{~Hz}$ per second; the final speed $\Omega=100 \mathrm{~Hz}$ is reached after 56 seconds; as can be seen, no tip-over phenomenon occurs during the process. This proves that if the installing height of the balancer $h_{b}$ equals $h_{u}$, the operating speed of the three-column centrifuge can be dramatically improved.

\subsection{Comparison between Dynamics of the Centrifuge before} and after the Balancer Is Installed. For a better understanding of the effect of the balancer, this section makes a simple comparison between dynamics of the centrifuge itself and those after the balancer is installed.

Before the balancer is installed, the eccentricity of the centrifuge $e$ equals $m_{u} r_{u} /\left(m_{u}+m_{c}\right)$ where $m_{u}$ denotes mass of the unbalance, $r_{u}$ is the gyration radius of the unbalance, and $m_{c}$ is the mass of the core assembly. After the balancer is employed, the eccentricity $e_{2}$ turns to $e_{2}=\left(m_{u} r_{u}-\right.$ $\left.2 m_{b} r_{b e}\right) /\left(m_{u}+2 m_{b}+m_{c}\right)$ where $m_{b}$ is mass of the balls and $r_{b e}$ is the equivalent gyration radius of the balls. As can be seen, if mass of the balls $m_{b}$ increases, $e_{2}$ could become smaller and 


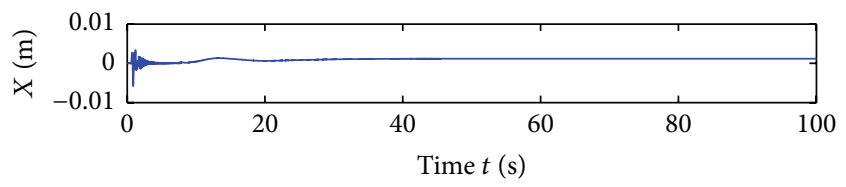

(a)

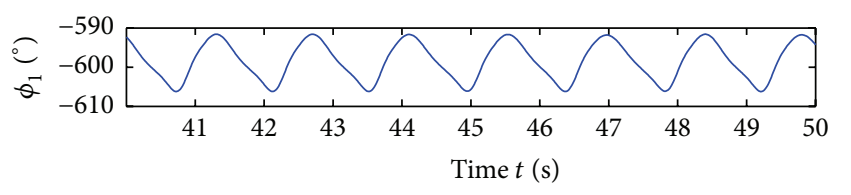

(c)

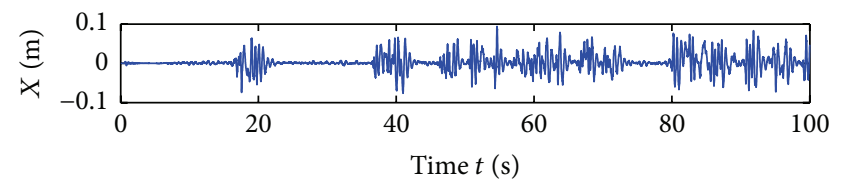

(b)

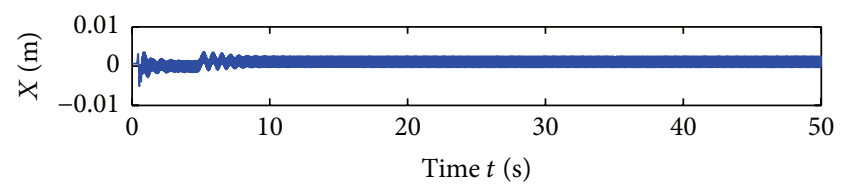

(d)

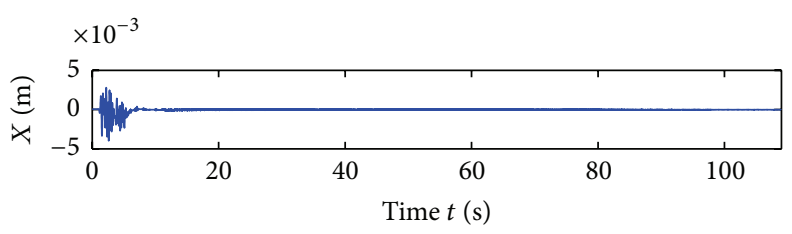

(e)

FIGURE 11: Validations of the tip-over speed $\Omega_{2}^{*}$ when $h_{b}=0.2 \mathrm{~m}, m_{b}=0.8 \mathrm{~kg}$, and $\Omega=11.5 \mathrm{~Hz}(\mathrm{a}) ; h_{b}=0.2 \mathrm{~m}, m_{b}=0.8 \mathrm{~kg}$, and $\Omega=12.5 \mathrm{~Hz}$ (b); $h_{b}=0.2 \mathrm{~m}, m_{b}=1.5 \mathrm{~kg}$, and $\Omega=10 \mathrm{~Hz}(\mathrm{c}) ; h_{b}=0.2 \mathrm{~m}, m_{b}=0.31 \mathrm{~kg}, \Omega=30 \mathrm{~Hz}$, and $\mu=0.3(\mathrm{~d}) ; h_{b}=0.1 \mathrm{~m}, m_{b}=1.5 \mathrm{~kg}$, and $\Omega=100 \mathrm{~Hz}(\mathrm{e})$.

smaller. And once $m_{b} \geq m_{b}^{*}$, an equilibrium between $2 m_{b} r_{b e}$ and $m_{u} r_{u}$ will happen which makes $e_{2}=0$. From this point of view, the balancer could reduce vibrations of the system directly.

We now compare the tip-over speeds. The tip-over phenomenon could still happen even after the eccentricity of the system is completely eliminated by the balancer. This is caused by the idea that the tip-over speed is not governed by equilibrium of the centrifugal forces; it is governed by the equilibrium of their corresponding moments relative to the suspension plane. In this situation, the installing height of the balancer $h_{b}$ plays a very important role. Taking the situation shown in Figure 10(c) for example, where $m_{u}=2 \mathrm{~kg}$, $h_{u}=0.1 \mathrm{~m}, h_{c}=0.03 \mathrm{~m}$, and $F_{\text {pre }}=100 \mathrm{~N}$ are considered, different values of $h_{b}$ could lead to different results: (1) if $h_{b}$ is smaller than $h_{u}$, the tip-over speed could be enhanced too, but the value of $\Omega_{2}^{*}$ is always limited; (2) if $h_{b}$ is bigger than $h_{u}$, there is an optimal mass of the balls $m_{b o}$ that could make $\Omega_{2}^{*}$ infinite; however, vibration of the system still exists because $m_{b o}$ is always smaller than $m_{b}^{*}$; (3) if $h_{b}$ equals $h_{u}$, as long as $m_{b} \geq m_{b}^{*}$, that is, as long as the eccentricity of the system is completely eliminated by the balancer, an infinite $\Omega_{2}^{*}$ could be achieved.

Side effects may appear after employing the balancer. The first one appears during the transient stage; as can be seen from Figure $8(d)$, if the positions of the balls and the unbalance are on the same side, the tip-over phenomenon could be induced more easily. The second one is caused by an inappropriate design of the balancer; as Figure 10(c) shows, if $h_{b}$ is higher than $h_{u}$ and a too big mass of the balls $m_{b} \geq m_{b}^{*}$ is employed, the tip-over speed could even be reduced.

Rolling friction acting on the balls may influence the performance of the balancer too. Although the balancer still works, its effect for counteracting the unbalance could be weakened. A detailed discussion of the imperfect location of the balls based on a planar rotor was made in [13] where countermeasures were also studied. To counteract the rolling friction, multiple balancers or a balancer containing multiple races might be adopted. For a three-column centrifuge, two balancers might be considered; the first one can be installed on the upper rim of the bowl and the second one may be fixed on the bottom rim. Theoretically, the effect of the two balancers is equivalent to that of the one fixed on the middle. However, it might have a better capability of coping with the rolling friction.

\section{Conclusions}

Modal analysis of a three-column centrifuge was carried out with two isolated natural frequency groups identified. The operating speed of the centrifuge should be higher than the highest frequency in the first group and lower than the smallest one in the second one.

Forces acting on the system at the steady state were studied. Based on the equilibrium of the centrifugal moments relative to the suspension system, the tip-over speed of the centrifuge without balancer was derived and then validated by simulations. The ideal operating speed of the centrifuge could be infinite if height of the unbalance equals that of the core assembly; however, due to instability caused by a small frictional coefficient in the spherical joints, the maximum operating speed of the centrifuge might be constrained.

Influences of the ball balancer on dynamics of the centrifuge were studied. If the installing height of the balancer $h_{b}$ is higher than that of the unbalance $h_{u}$, there is an optimal mass of the balls that could keep the operating speed infinite. If $h_{b}$ is smaller than $h_{u}$, no matter how the balancer is 
designed, the maximum operating speed of the centrifuge is always limited. The ideal condition is $h_{b}=h_{u}$; as long as the mass of the balls is bigger than or equal to $m_{b}^{*}$, the operating speed can be infinite.

Rolling friction acting on the balls may have negative effect on the performance of the balancer. Some future works have to be carried out on how to cope with it. A possible way is employing two balancers: one on the top side of the bowl and the other one on the bottom side. It might have a better capability of coping with the rolling friction; however, its performance still needs to be proved by experiments.

\section{Conflict of Interests}

The authors declare that there is no conflict of interests regarding the publication of this paper.

\section{Acknowledgments}

The authors would like to acknowledge the support by China Postdoctoral Science Foundation (2013M531271), National Natural Science Foundation of China (51205166), National Natural Science Foundation of China (51575236), and China Scholarship Council (201308320011).

\section{References}

[1] E. G. Gerke and V. A. Noskov, "Mechanization of bottomdischarge three-column centrifuges," Chemical and Petroleum Engineering, vol. 1, no. 7, pp. 573-574, 1965.

[2] E. G. Gerke, "Mechanized hydraulic-drive three column centrifuge," Chemical and Petroleum Engineering, vol. 3, no. 3, pp. 185-186, 1967.

[3] I. N. Il'yashenko and E. G. Gerke, "Continuous three-column centrifuge for deoiling metal chips," Chemical and Petroleum Engineering, vol. 4, no. 4, pp. 279-280, 1968.

[4] V. G. Likuchev, "Calculating the frequencies of natural vibrations and the amplitudes of forced vibrations in three-column centrifuges," Chemical and Petroleum Engineering, vol. 3, no. 1, pp. 6-14, 1967.

[5] V. G. Likuchev, "Rigidity of supports in three-column centrifuges," Chemical and Petroleum Engineering, vol. 4, no. 10, pp. 799-802, 1968.

[6] B. A. Vanichkin, "Investigation of the steady motion of the elastically suspended mass of three-column centrifuges," Chemical and Petroleum Engineering, vol. 6, no. 8, pp. 632-635, 1970.

[7] S. Bae, J. M. Lee, Y. J. Kang, J. S. Kang, and J. R. Yun, "Dynamic analysis of an automatic washing machine with a hydraulic balancer," Journal of Sound and Vibration, vol. 257, no. 1, pp. 318, 2002.

[8] H.-W. Chen and Q.-J. Zhang, "Stability analyses of a vertical axis automatic washing machine with a hydraulic balancer," Mechanism and Machine Theory, vol. 46, no. 7, pp. 910-926, 2011.

[9] L. Urbiola-Soto, M. Lopez-Parra, and F. Cuenca-Jimenez, "Improved design of a bladed hydraulic balance ring," Journal of Sound and Vibration, vol. 333, no. 3, pp. 669-682, 2014.

[10] C.-H. Jung, J.-T. Kim, and Y.-H. Choi, "A computational study on the flow characteristics of a self-compensating liquid balancer," Journal of Mechanical Science and Technology, vol. 25, no. 6, pp. 1465-1474, 2011.
[11] H.-W. Chen and Q.-J. Zhang, "Stability analyses of a vertical axis automatic washing machine without balancer," Journal of Sound and Vibration, vol. 329, no. 11, pp. 2177-2192, 2010.

[12] Y. P. Smirnov, "Some frictional effects in a spherical joint," Soviet Applied Mechanics, vol. 17, no. 10, pp. 911-915, 1981.

[13] Y. Ishida, T. Matsuura, and X. L. Zhang, "Efficiency improvement of an automatic ball balancer," Journal of Vibration and Acoustics, vol. 134, no. 2, Article ID 021012, 2012.

[14] C.-H. Hwang and J. Chung, "Dynamic analysis of an automatic ball balancer with double races," JSME International Journal, Series C: Mechanical Systems, Machine Elements and Manufacturing, vol. 42, no. 2, pp. 265-272, 1999.

[15] K. Green, A. R. Champneys, and N. J. Lieven, "Bifurcation analysis of an automatic dynamic balancing mechanism for eccentric rotors," Journal of Sound and Vibration, vol. 291, no. 3-5, pp. 861-881, 2006. 


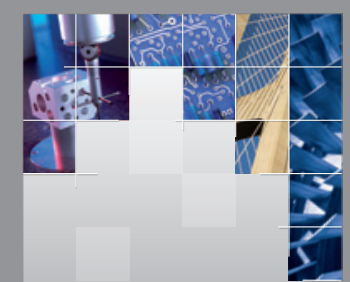

\section{Enfincering}
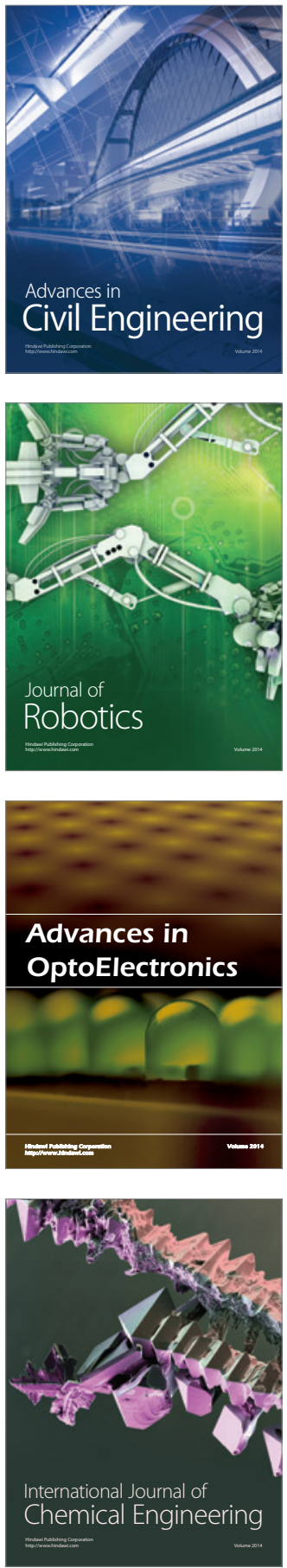

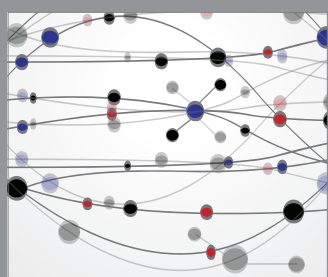

The Scientific World Journal

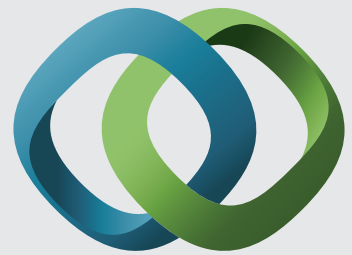

\section{Hindawi}

Submit your manuscripts at

http://www.hindawi.com
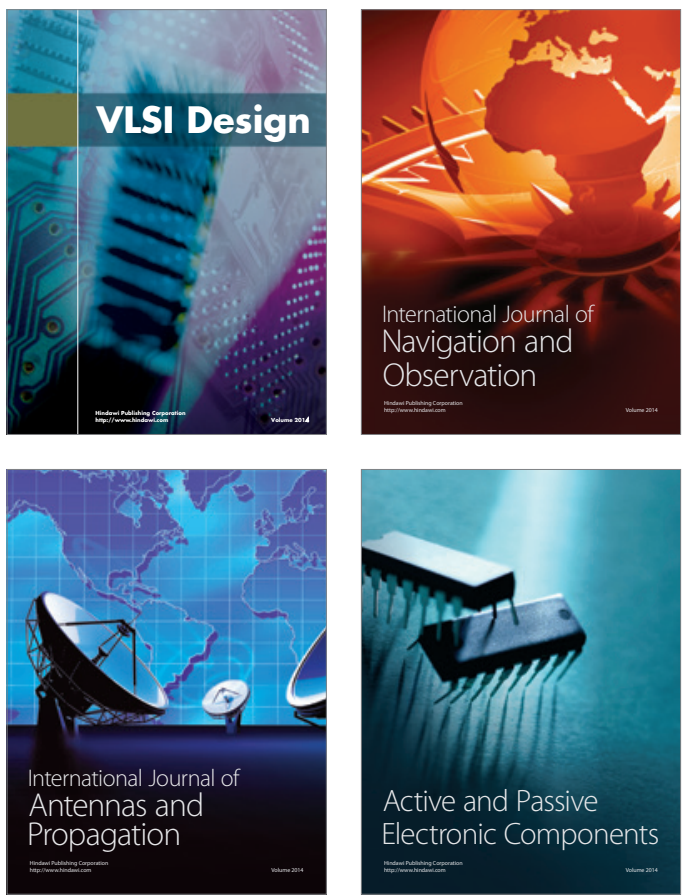
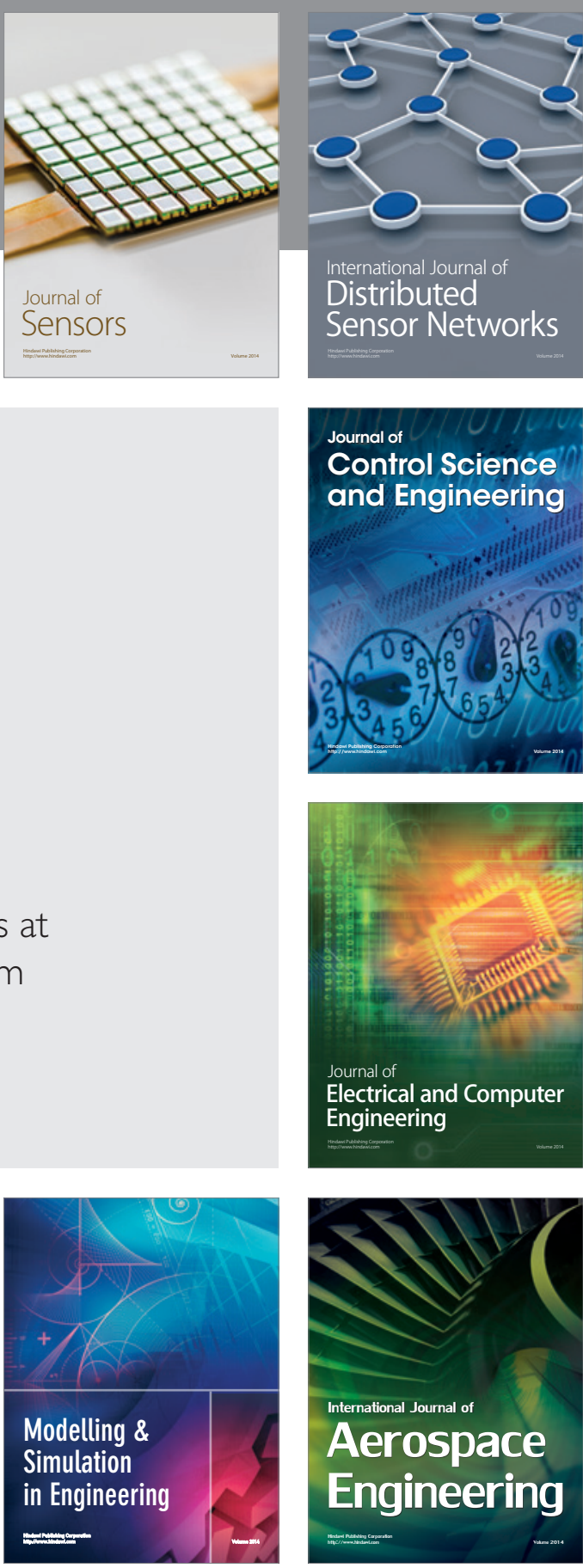

International Journal of

Distributed

Sensor Networks

Journal of

Control Science

and Engineering
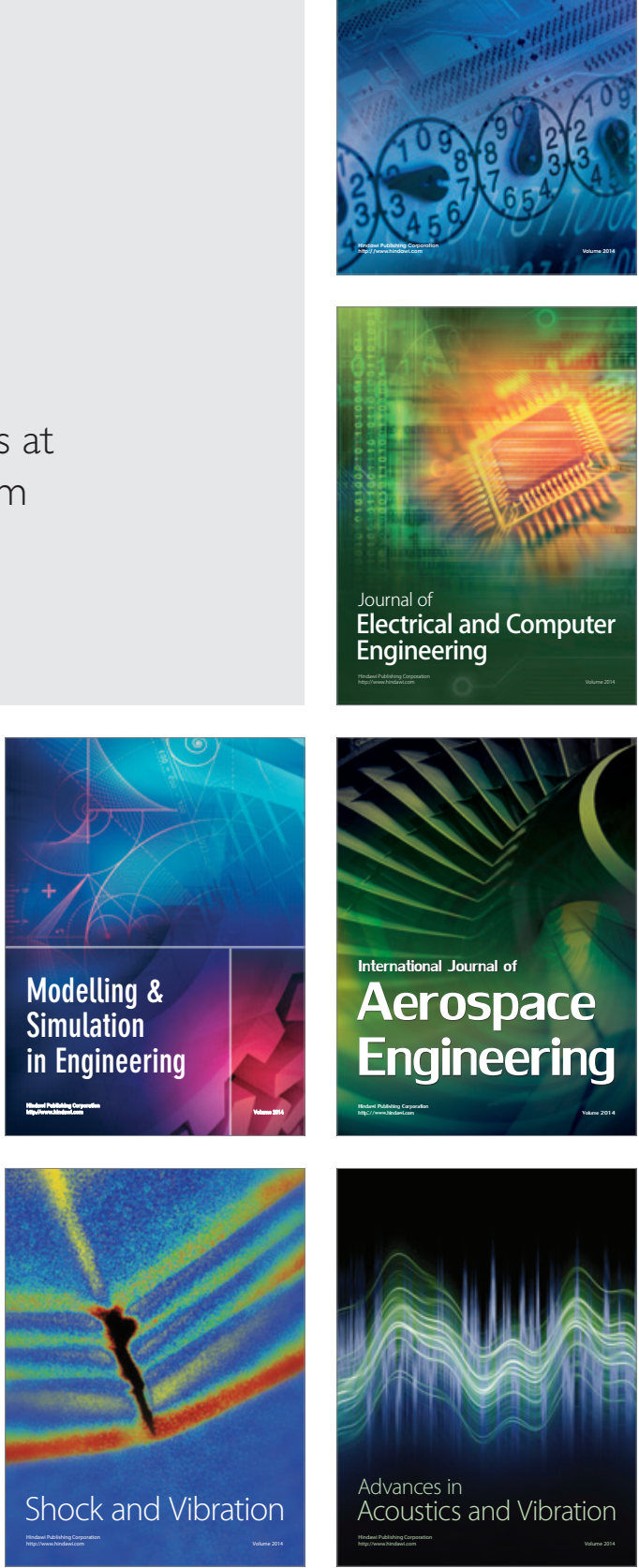